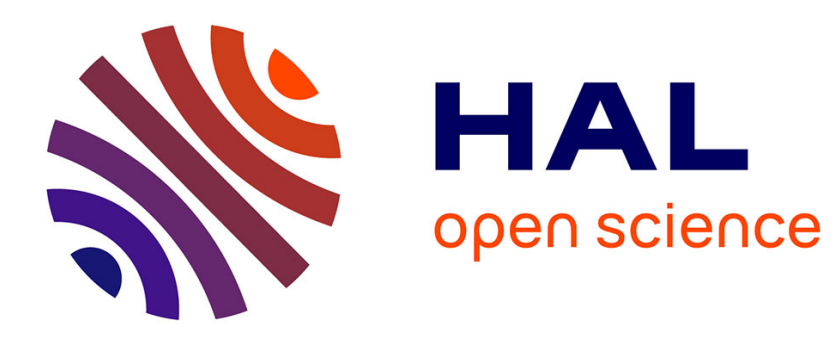

\title{
Sans la Vierge, Richard sans Peur et la femme Denis Hüe
}

\section{To cite this version:}

Denis Hüe. Sans la Vierge, Richard sans Peur et la femme. Annales de Normandie, 2014, Richard sans peur, duc de Normandie, entre histoire et légende, 64 (1), pp.171-188. 10.3917/annor.641.0173 . hal-02458055

\section{HAL Id: hal-02458055 https://hal.univ-rennes2.fr/hal-02458055}

Submitted on 28 Jan 2020

HAL is a multi-disciplinary open access archive for the deposit and dissemination of scientific research documents, whether they are published or not. The documents may come from teaching and research institutions in France or abroad, or from public or private research centers.
L'archive ouverte pluridisciplinaire HAL, est destinée au dépôt et à la diffusion de documents scientifiques de niveau recherche, publiés ou non, émanant des établissements d'enseignement et de recherche français ou étrangers, des laboratoires publics ou privés. 


\section{SANS LA VIERGE, RICHARD SANS PEUR ET LA FEMME}

\section{Denis Hüe}

Association Les Annales de Normandie | « Annales de Normandie »

2014/1 64e année | pages 173 à 188

ISSN 0003-4134

ISBN 9782902239313

\section{Article disponible en ligne à l'adresse :}

https://www.cairn.info/revue-annales-de-normandie-2014-1-page-173.htm

Distribution électronique Cairn.info pour Association Les Annales de Normandie.

(C) Association Les Annales de Normandie. Tous droits réservés pour tous pays.

\section{(cc) BY-NC}




\title{
Sans la Vierge, Richard sans Peur et la femme
}

\author{
DenIs HÜE*
}

$\mathrm{E}$ N PROPOSANT une contribution sur Richard et la Vierge, j'avais en tête cette anecdote qui lui est rattachée : le miracle du clerc fornicateur, habituellement miracle marial, dans lequel Richard se présente comme un intrus. Confiant dans ma bonne étoile et dans l'omniprésence avérée de la sainte Vierge dans la littérature médiévale, et particulièrement normande, j'étais sûr de trouver un corpus significatif de textes, miracles, allusions, références implicites ou explicites permettant de mettre en perspective la biographie de Richard revisitée pour l'occasion par de pieux chroniqueurs et la fête aux Normands, ou tout au moins ses prodromes et ses implicites.

Il se trouve que mes recherches successives se sont soldées par des échecs multiples ; il n'y a vraiment pas grand-chose de marial dans la biographie de Richard, qu'il s'agisse de son existence réelle ou des récits qui en ont été tirés : peu d'invocations à la Vierge dans les discours qui lui sont attribués, peu de dévotions à Notre Dame, si ce n'est, malgré tout, dans les cathédrales, pas de merveilleux marial, sous aucune forme.

Néanmoins, c'est le privilège du chercheur, une enquête, si elle semble se solder par un échec, n'en est pas moins riche d'enseignements : c'est sur ceux-là qu'il importera tout d'abord de s'attarder. Pourquoi aussi peu de dévotion mariale chez Guillaume de Jumièges et Dudon de Saint-Quentin ? Quels sous-entendus évidents - et moins évidents peut-être - cette absence laisset-elle deviner ? Dans un deuxième temps, il faudra bien s'attacher un peu à la dimension mariale de l'épisode du clerc fornicateur : pourquoi Wace s'encombre-t-il d'un tel artefact, si ce n'est pour des raisons plus localistes encore que mariales - alors même que son auditoire sait parfaitement à quoi s'en tenir ? $\mathrm{Ou}$, symétriquement, que vient faire cet épisode rouennais dans la collection des miracles associés dès le début à la Conception de la Vierge ? Cela devrait permettre, pour finir, de s'interroger non plus sur l'élément lui-même, déjà bien balisé, mais sur sa place dans l'économie du Roman de Rou, et dans la fabrication de la légende des ducs de Normandie.

* Université de Rennes II, Centre d'Étude des Textes Médiévaux-Centre d'Études des Langues et Littératures Anciennes et Modernes (CETM-CELLAM, EA 3207), denis.hue@wanadoo.fr. 
Le premier élément qui retiendra notre attention est évidemment le travail de christianisation opéré sur la figure de Richard ; on sait combien le mot de martyr est utilisé pour qualifier la figure de Guillaume Longue Épée, à la fois tué traîtreusement et porteur symboliquement de la cuculle monastique ; il s'agit maintenant de faire de Richard une autre figure de la sainteté, saint évangélisateur - une autre figure du martyre - étymologiquement le témoignage - en cela qu'il invite à la conversion tous ceux qu'il rencontre, ici les princes danois, de façon presque miraculeuse ${ }^{1}$. Dans cette démarche, la référence à la personne de la Vierge pourrait sembler évidente : ce qui est frappant au contraire, c'est sinon son absence, du moins une présence très minimale, puisqu'on reprend, presque mot pour mot, les expressions du Credo ${ }^{2}$. Le jeune Richard s'exerce à l'éloquence, se consacre à Dieu, non pas en se confiant à la Vierge auxiliatrice, mais dans une démarche volontariste et vigoureuse :

"Vivaci lepore affluenter armabat linguam, facundaeque ubertatis colloquio insignibat eam. Peragrabat studio et retractabat quae ignorabat, nec abdebantur ei quae sunt obscura. Pueritiae suae aetatulam Jesu Christo consecrabat, seque totum divinis praeceptis, tenerae adhuc licet aetatis esset, mancipabat » ${ }^{3}$.

Quand il est question de faire l'apologie du duc, dans une admirable conclusion, c'est en suivant les versets des Béatitudes ${ }^{4}$ que Dudon articulera son propos : nulle part une invocation à la Vierge, nulle part ou presque une mention de la mère de Dieu, et cela dans l'ensemble de l'œuvre de Dudon. Elle n'est mentionnée qu'à propos de Rollon et de la tunique de la Vierge à Chartres ${ }^{5}$, puis au moment du baptême de Rollon : après s'être enquis des églises et des saints particulièrement vénérés sur ses terres et sur les terres voisines, il décide de leur faire don de divers domaines :

"Antequam diuidatur terra meis principibus Deo et sanctae Mariae sanctisque denominatis desidero partem istius terrae dare ut dignentur mibi in auxilium subuenire $"^{6}$.

Il s'agit symboliquement, en leur donnant des fiefs, de se les assujettir et de faire de Denis, le saint des Francs, comme de Marie, dont il a pu expérimenter

1 Voir L. Mathey-Maille, Écritures du passé, histoires des ducs de Normandie, Paris, Champion, 2007, p. 133-134, et plus précisément, p. 208-214. Cette proclamation de la foi rapproche Richard d'une autre forme de sainteté, celle du confesseur.

2 "ex sancrosanctae Virginis carne, angelo nuntiante, processit in carnem... " : Dudon DE SAInTQuentin, De moribus et actis primorum Normanniae ducum, J. Lair (éd.), Caen, F. Le Blanc-Hardel, 1865 (ci après Dudon, De moribus), p. 284.

3 Ibid., p. 230. " Il avait l'expression vive, tout ornée de grâce, et il la rehaussait par la pratique d'une conversation riche et abondante. Il parcourait avec ardeur et révisait ce qu'il ignorait, et les choses obscures ne lui restaient pas cachées; il consacrait les jeunes années de son enfance à Jésus-Christ et il se consacrait tout entier aux préceptes divins, bien qu'il fût encore d'un âge tendre ».

4 Mt 5, 3-12.

5 "Gualtemus episcopus, quasi missam celebraturus infulatus, bajulans crucem atque tunicam sacrosanctae Mariae Virginis in manibus [...] paganorum terga velis verberat et mucronibus. " (Dudon, De moribus, p. 162).

6 Ibid., p. 170 : «Avant de diviser ma terre entre mes princes, je souhaite donner à Dieu, à sainte Marie et aux saints mentionnés une part de cette terre, pour qu'ils daignent me venir en aide ». 
la puissance à Chartres, ses débiteurs. On comprend à ce moment que toutes les cathédrales Sainte-Marie de Rouen, de Bayeux et d'Évreux soient richement dotées ; il s'agit bien pour Rollon de se les inféoder, Marie peut être dangereuse, tout comme saint Denis à qui Rollon restitue Berneval7.

La dernière occurrence du nom de Marie se trouve au moment où on ensevelit Guillaume Longue Épée :

"et Rotomagensi urbi cum magno ejulatu delatum in ecclesiam beatae Mariae genitricis Dei honorifice sepelierunt $»^{8}$.

Il ne s'agit pas alors d'une personne, mais d'un nom de lieu. Des recherches sur le mot Virgo et sa famille nous montreront qu'il est employé pour exalter l'âme pure de Richard, mais jamais pour parler de la Vierge, jusqu'à la mort du duc inclusivement.

À l'époque de Richard, comme au moment où écrit Dudon, quelques années après, il n'y a pas de fête aux Normands : la Vierge, si elle a une importance, est exclusivement mère de Dieu, dédicace de sanctuaire, relique chartraine plutôt à craindre, et n'entre pas dans l'univers très viril des seigneurs vikings. Mais à y réfléchir, le grand initiateur de la dévotion mariale après l'an Mil, Fulbert de Chartres, est encore vivant, et l'Occident commence à peine à découvrir ce pan de la foi chrétienne'. Dudon et Guillaume de Jumièges parleront de leurs ducs sans jamais ou presque invoquer la figure de la Vierge, parce que cela n'a pas lieu d'être jusqu'à la fin du XI' siècle.

Il n'en est pas de même à la fin du XII ${ }^{\mathrm{e}}$ siècle. Il est difficile de déterminer le moment de la rédaction de la Conception Nostre Dame par Wace : on a longtemps cru que les textes hagiographiques du poète étaient des œuvres de jeunesse, sans prendre en compte la dimension politique de ces œuvres, qui concourent à établir une présence normande dans les choses de la foi. On admet que ce texte n'a pu être écrit avant 1130 ; il rapporte, dès ses premières lignes, comment Guillaume est l'instaurateur de la fête de la Conception en Normandie et son restaurateur en Angleterre, sous l'impulsion d'Helsin, abbé de Ramsay $^{10}$, avant de rapporter le récit de la Conception de Marie, suivant

7 François Neveux m'a soufflé que toutes les abbayes richement dotées étaient, fortuitement, celles qui étaient sous la dépendance directe de l'archevêque de Rouen.

8 Dudon, De moribus, p. 208 : «Et à la ville de Rouen, une fois porté avec de grandes lamentations dans l'église de la bienheureuse Marie mère de Dieu, on l'ensevelit avec tous les honneurs ».

9 Voir entre autres Marie. Le culte de la Vierge dans la société médiévale, D. Iogna-Prat, E. Palazzo et D. Russo (dir.), Paris, Beauchesne, 1996, ainsi que M. Rubin, Mother of God, a bistory of the Virgin Mary, Londres, Allen Lane, 2009.

10 Pour plus de détails sur cet aspect, je renvoie à mon " Guillaume le Conquérant et la Vierge », dans H. Legros (dir.), Guillaume le Conquérant face aux défis, Orléans, Paradigme, Medievalia 66, 2008, p. 69-91. Sur la dévotion à la Conception de Marie au cours du Moyen Âge en Normandie, voir la première partie de ma thèse, La Poésie Palinodique à Rouen, 1486-1550, Paris, Éditions Champion (Bibliothèque littéraire de la Renaissance, série III, tome XLIV), 2002, p. 32-216. Pour une approche plus large, F. THELAmon (dir.), Marie et la "Fête aux Normands». Dévotion, images, poésie, Rouen, PURH, 2011. 
en cela les évangiles apocryphes. La première attestation du miracle d'Helsin se trouve dans l'œuvre d'Eadmer et ouvre son Sermo de conceptione ${ }^{11}$, premier texte à rendre compte des marques de la dévotion populaire en milieu anglonormand. À ce miracle s'ajoute l'histoire du Diacre de Hongrie, dans lequel un jeune homme pétri de dévotion mariale quitte sa maison le soir de ses noces pour mieux se consacrer à la Vierge, devenant par la suite évêque d'Aquilée. Le projet d'Eadmer, après avoir montré la fondation anglaise de la dévotion et souligné son origine orientale, est cependant de prouver que cette ferveur est partagée également entre Angleterre et Normandie ; c'est ce qu'apporte le miracle suivant où un chanoine, prêtre de son ordre, dans une région proche du littoral - pelago gallico - succombe au désir de fornication et s'apprête à traverser la Seine a villa quadam ubi cum uxore alterius fornicatus fuerat.

«Dans une région proche du littoral, un chanoine, ordonné prêtre, habitué à chanter les heures de la bienheureuse Vierge Marie, rentrant d'une maison où il avait forniqué avec l'épouse d'autrui, revenant à la ville dans laquelle il était attendu et s'appliquant à la rejoindre, désirant traverser la Seine, monté seul dans une barque, il commença à chanter les heures de la Vierge pendant qu'il naviguait. Quand il en était à l'invitatoire Ave Maria gratia plena Dominus tecum, et qu'il se trouvait au milieu du fleuve, voici qu'une grande foule de démons le précipita avec sa barque dans les profondeurs de l'onde, et s'empara de son âme pour la tourmenter.

$\mathrm{Au}$ troisième jour, dans le lieu où les démons le tourmentaient par leurs tortures, voici venir la mère de Jésus avec une grande foule d'anges, demandant :

"Pourquoi faites-vous injustement souffrir l'âme de notre serviteur?

- Elle nous revient de droit, et à juste titre, car elle a été prise alors qu'il était dans nos œuvres".

La mère de Jésus leur répondit :

"Si elle doit revenir à celui dont il accomplissait les œuvres, elle doit être nôtre, car il chantait nos matines quand vous l'avez fait périr : vous êtes davantage coupables vous qui avez agi envers moi de façon inique".

À ces mots les démons s'enfuirent en tous sens, la bienheureuse Marie reconduisit l'âme à son corps et ramenant par le bras l'homme rappelé du tombeau, ordonnant à l'eau de se dresser comme un mur à gauche et à droite, le mena sain et sauf du fond de l'abîme au port $\aleph^{12}$.

11 J.-P. Migne, Patrologie latine (désormais P.L.), t. 159, col. 319. L'attribution de ce texte à Eadmer est évidemment sujette à caution, mais sera maintenue pour des raisons pratiques; pour ces premières controverses conceptionnistes, on se référera à M. LAMY, L'Immaculée Conception. Étapes et enjeux d'une controverse au Moyen Âge (XII $-X V^{e}$ siècles), Paris, IEA, 2000, qui ne semble pas avoir été informée de mes travaux sur ces textes.

12 P.L., t. 159, col. 321 : In pelago Gallico canonicus quidam, ordine sacerdos, solitus B. Mariae Virginis horas canonicas decantare, a villa quadam, ubi cum uxore alterius fornicatus fuerat, rediens, ad oppidum in quo morabatur tendere curans, et pelagus Sequanae transmeare cupiens, solus navem ingressus Dominicae Matris horas navigando canere coepit. Cumque invitatorium, Ave, Maria, gratia plena, Dominus tecum, diceret, et jam in medio fluminis esset, ecce turba magna daemonum in profundo pelagi, una cum ejus navicula eum praecipitavit, 
Éperdu de reconnaissance, le clerc demande ce qu'il peut faire en action de grâce : la Vierge lui demande évidemment de ne plus pécher, et de célébrer la fête de la Conception ${ }^{13}$; ce miracle est essentiel dans le projet d'Eadmer, car il propose un ancrage normand de la dévotion, qui permet d'articuler une symétrie avec la dévotion strictement anglaise qu'impliquait le récit d'Helsin.

En même temps, on me permettra de souligner les éléments qui font de ce récit une construction textuelle bien plus qu'un témoignage local : lui qui sera bientôt localisé très précisément à Saint-Ouen est pour le moment dans un lieu indéterminé. Le seul ancrage normand se fait autour de la Seine, que l'on traverse en barque, et qui est assez large pour que le terme de pelagus lui soit affecté. On pense évidemment à Rouen et son abbaye de Saint-Ouen plus qu'à Jumièges ou Saint-Wandrille, qui ne sont pas assez proches du fleuve; la rive sud de Rouen est assez peu peuplée alors, et il semble bien étrange qu'un clerc ait besoin de traverser le fleuve pour assouvir son désir ; il semble plus étrange encore qu'il soit à même de le faire en naviguant seul pour traverser deux cents mètres environ, en pleine nuit de surcroît. On voit bien que le motif est clairement légendaire à ses débuts, même s'il s'ancrera contre toute vraisemblance dans une topographie normande.

Quelques motifs sont d'ailleurs assez explicites et montrent la reconstruction du récit : au-delà du motif initial d'une simple noyade et d'une résurrection, le narrateur ajoute des éléments comme les trois jours de tourment subis par le clerc, qui sont à la fois figure d'un châtiment purgatoire et écho des trois jours de la mort du Christ ; la remontée du fond des flots où la Vierge commande aux flots de s'écarter, aquam a dextris et a sinistris quasi murum stare jubens, ressemble à la traversée de la Mer Rouge, et par cela à l'élection d'un nouveau peuple, à une figure du baptême. Symboliquement, c'est bien au travers du clerc la création d'une nouvelle communauté réunie par une même dévotion à la Vierge qui est affirmée.

et ejus animam rapuit ad tormenta. Die autem tertia locum quo daemones eum tormentis afficiebant, venit Mater Jesu cum magna angelorum societate, dicens eis : Ut quid animam famuli nostri ita injuste affligitis? Nos, inquiunt, eam debemus habere, et merito, quoniam in nostris operibus capta est. Quibus Mater Jesu: Si illius debet esse cujus opera faciebat, ergo nostra debet esse, quoniam matutinas nostras, dum vos eum peremistis, decantabat; unde magis rei estis, qui iniqua contra me egistis. His dictis daemones huc illucque dispersi fugerunt, et beata Maria animam reduxit ad corpus, et hominem ab utroque funere suscitatum per brachium arripiens, aquam a dextris et a sinistris quasi murum stare jubens, de profundo pelagi ad portum incolumem reduxit.

13 Directement après la citation précédente : At ille gaudens pedibus B. Virginis prostratus ait: Charissima domina mea, et Virgo speciosa Christo gratissima, quid tibi tribuam pro tantis beneficiis quae mibi fecisti? Liberasti me de ore leonis, et de tormentis inferni gravissimis animam meam. Cui Mater Jesu ait: Precor te, ne de caetero in adulterii peccatum cadas, ne fiat tibi novissimus error pejor priore. Precor te iterum ut de caetero festum conceptionis meae devote celebres annuatim VI Idus Decembris, et ubique celebrandum praedices. Mox ut id dixit B. Virgo Maria, eo cernente coelos ascendit, et ipse eremiticam vitam ducens, quod sibi acciderat, cunctis audire volentibus narravit, et postea quandiu vixit, illud festum conceptionis solemniter et devote celebravit, et celebrandum praedicavit. 
J'ai déjà étudié ailleurs ce miracle ${ }^{14}$, qui s'inscrit dans une constellation de versions où se multiplient les variantes : le clerc meurt en chantant ou non les louanges de Marie, il ressuscite ou ne ressuscite pas - dans ce cas, c'est un phylactère contenant la prière mariale ou un lys qui sort de sa bouche et autorise l'inhumation en terre sacrée. Retenons cependant que le texte d'Eadmer est un des plus anciens de cette vaste nébuleuse qui s'étend dans toute la littérature mariale européenne aux $\mathrm{XII}^{\mathrm{e}}$ et $\mathrm{XIII}^{\mathrm{e}}$ siècles. Nous le voyons apparaître avec des variantes chez Guillaume de Malmesbury ${ }^{15}$, qui supprime la dimension conceptionniste, mais garde la proclamation de l'Ave Maria, et de là, avec de substantielles variantes, chez Adgar ${ }^{16}$ et Nigel de Longchamps ${ }^{17}$, qui sont postérieurs à Wace ; ce sont la localisation, l'état du pécheur - est-il noyé après le péché ou avant son intention - et les modalités de sa résurrection qui varient ${ }^{18}$. Le miracle est donc dans sa première version un récit conceptionniste, issu d'un milieu anglo-normand, et sert en cela des desseins particuliers, que les versions plus tardives (je pense à celle de Gautier de Coinci par exemple) pourront gommer sans remords. Ce qui retiendra notre attention ici, c'est bien sûr la parenté de ce miracle marial, présent dans le premier quart du XII siècle dans l'œuvre d'Eadmer, avec celui qui figure dans les anecdotes consacrées à Richard : deux versions en sont présentes, chez Wace et chez Benoît, et elles rapportent à peu près la même histoire, à cela près que Benoît, plus loquace, donne une multitude de détails ; ainsi, il s'agit d'un sacristain chez Wace : « out a cel tens uns secrestein, / Tenuz esteit pour leal muine $»^{19}$, qui devient chez Benoit « segrestein, custode e garde e marrugler : les choses gardoet deu moster $»^{20}$ : sacristain, custode et marguillier renvoient à des fonctions différentes en principe ${ }^{21}$, et les expressions ne sont pas exactement synonymes. On voit par cet exemple comment Benoît s'efforce d'amplifier le texte de Wace l'augmentatio est une figure de style digne de l'auctor - sans forcément ajouter de réelle information.

14 Voir D. Hü E, La Poésie palinodique à Rouen, 1486-1550, Paris, Champion, 2002, p. 159-165; et mon " Guillaume le Conquérant et la Vierge », op. cit., p. 88-91.

15 J.-M. CAnal, El libro "De laudibus et miraculis Sanctae Mariae" de Guillermo de Malmesbury, OSB (tc. 1 143) Estudio y texto, Rome, Alma Roma Libreria Editrice, 1968, p. 98-99.

16 Adgar, Le Gracial, publié par Pierre Kunstmann, Ottawa, Éditions de 1'Université d'Ottawa (Publications médiévales de l'Université d'Ottawa / University of Ottawa Mediaeval Texts and Studies, 8), 1982, p. 67-72.

17 Nigel of Canterbury, Miracles of the Virgin Mary, in verse, miracula sante Dei genitricis Virginis Marie, versifice, J Zıоцкоwsкi (éd.), Toronto Medieval latin texts, Pontifical Institute of Medieval studies, 1986, p. 39-45.

18 On se référera ici, plus qu'à Mussafia, qui ne dit pas grand-chose sur ce miracle, à l'article de H. KJellman, «Sur deux épisodes de Gautier de Coincy ", Romania, t. XLVII, 1921, p. 588-594.

19 Wace, Roman de Rou, A. J. Holden (éd.), Paris, SATF, 1970-1973, t. I, Troisième Partie (notée désormais III), vers 348-349.

20 Benoît de Sainte-Maure, Chronique des ducs de Normandie, C. Fahlin (éd.), Almqvist, t. II, vers 27 634-27 636 .

21 Le custode est chargé du soin des ornements sacerdotaux, le marguillier a l'administration des affaires temporelles d'une église, il peut être assimilé au bedeau ou au sonneur. 
On retrouve dans cette version l'abbaye Saint-Ouen, et la proximité est grande avec le Robec, le petit affluent de la Seine qui coule non loin de l'abbaye. S'il y a resserrement et gain en vraisemblance au moins sur le plan topographique, il est difficile en revanche de croire que l'on peut se noyer dans le Robec : ce petit cours d'eau aujourd'hui couvert est décrit par Farin dans son Histoire de Rouen comme régulièrement ensabléz ${ }^{2}$, et on le traverse, nous dit le texte de Wace, par une planche. Il y a de fait aussi peu de vraisemblance de l'un à l'autre et, quelle que soit la volonté d'ancrage dans une topographie locale, elle relève pour l'essentiel d'un artifice rhétorique.

Si dans la version initiale, les choses sont équilibrées entre un péché avéré et la dévotion (le clerc a succombé, mais il prononce la prière mariale au moment de se noyer), dans les versions richardiennes (où ce n'est pas un clerc, mais un moine qui est en cause), elles le sont parce que le péché reste en intention et n'est pas encore consommé. La noyade du moine est directement suivie chez Wace d'une dispute entre ange et démon; mais les arguments de ce dernier sont très proches de ceux qu'avait exposés Eadmer :
«Deables dist : "tu me faiz tort,
Que me touz l'alme que j’en port ;
Donc ne sez tu ke l'alme est meie
Des qu'ele est prise en male veie?
En male veie esteit entree
E en mal ovre l'ai truvee,
En veie de mal s'esteit mise,
E en veie de mal l'ai prise" ${ }^{23}$.

À défaut d'avoir effectivement succombé à la tentation, le moine est « en male veie », et en "male ovre ». Comme il n'est pas question dans cette version de faire intervenir la Vierge, le moine n'a pas chanté d'Ave Maria ; c'est ce qui justifie l'emploi par l'ange d'une autre argumentation :
«Ne encore n’ad il fait le pechié
Dunc tu l'as ja pris e jugié.
De l'abeïe esteit eissuz
E a la planche esteit venuz,
Uncor se peüst il retraire
S'il ne chaïst, del pechié faire $»^{24}$.

En symétrique de la controverse sur l'absolution avant le péché ${ }^{25}$ se met en place un débat sur la damnation avant celui-ci. C'est alors qu'est proposé le recours à Richard :

22 F. Farin, Histoire de la ville de Rouen, Rouen, Le Brun, 1738, t. I, p. 12-13.

23 Roman de Rou, III, vers 385-392.

24 Ibid., vers $415-420$.

25 Évoquée entre autres chez Dante, Enfer XXVII : «ch'assolver non si può chi non si pente, / né pentere e volere insieme puossi / per la contradizion che nol consente». 


\begin{abstract}
«Alum ça al conte Richart
Si nus metum en son esgart,

il nus jugera leaument,

Qu'il ne fait nul faus jugement $»^{26}$.
\end{abstract}

Il y a, objectivement, à s'étonner de ce qu'un mortel soit saisi comme arbitre d'une cause qui intéresse le monde céleste, et c'est plutôt le contraire qui arrive en général : l'ambition est de faire de Richard un rex justitiae, équivalent de Melchisedech ${ }^{27}$ et de Salomon. On connait la suite : Richard propose de replacer le moine sur sa planche, juste avant sa chute, et d'observer ce qui se passe. Évidemment, le moine repart en arrière :

«Des que li moines se aparçut

E sur la planche en pais s'estut,

Ariere mist plus tost sun pié

Ke hoem ki a serpent marchié ${ }^{28}$.

Ce dernier vers ne doit pas être compris simplement comme une notation réaliste : le fait de marcher sur un serpent nous rapproche du geste fondateur de la nouvelle Ėve, qui écrase le Malin de son talon, et c'est comme un écho à une des formules favorites de la foi conceptionniste qui se manifeste ici. La figure de Richard comme nouveau Salomon ("Qu'il ne fait nul faus jugement ») nous montre s'il en était besoin que le texte est plein d'arrière-pensées : ce qui est en jeu ici, c'est bien sûr d'une part la question du libre arbitre, celle du Salut et du péché, de l'autre la fondation d'une tradition locale, d'une foi liée au pouvoir et ancrée dans le royaume anglo-normand, qu'il s'agisse de Guillaume le Conquérant fondateur d'une dévotion conceptionniste avec l'abbé de Ramsay ou d'un Richard bienfaiteur du monastère de Saint-Ouen.

Nous trouvons dans les deux versions de ce miracle la même problématique d'une publicité donnée à l'événement ; cet élément permet de quitter le statut du récit merveilleux et édifiant pour devenir aussi bien un témoignage historique qu'une vérité de la foi proclamée solennellement par la Vierge elle-même, destinée à fonder une dévotion. Dans les deux cas, il s'agit de témoigner à la fois de la faute accomplie ou projetée, et du rachat qui a été obtenu par faveur surnaturelle et par justice. Le clerc / moine, dans les deux versions, avoue sa faute et la raconte à la ronde :

"et ipse eremiticam vitam ducens, quod sibi acciderat, cunctis audire volentibus narravit $»^{29}$.

26 Roman de Rou, III, vers 429-432.

27 C'est ainsi que son nom est glosé : Melchisedech qui interpretatione latina dicitur rex justitiae, rex pacis, Ambroise, De Abraham, P.L. 14, col. 427.

28 Roman de Rou, III, vers 470-474.

29 P.L., t. 159, col. 321 : «Et lui-même, menant la vie d'un ermite, racontait ce qui lui était arrivé à tous ceux qui souhaitaient l'entendre ». 
Chez Wace, la confession au duc et à l'abbé fait place à un récit assez connu pour donner naissance à une locution : «al passer planche vus gardez $»^{30}$. La reconnaissance du clerc se porte pour l'un sur la Vierge, à l'égard de qui il s'engage (c'est la fonction du récit étiologique) à célébrer la fête de la Conception, pour l'autre sur Richard qui devient une figure surnaturelle, capable de délivrer le moine des griffes du diable :

«Cument ala, cument peri, cument diables l'enginna, cument li quens le delivra: Tote la verité cunta... »"

Dans les deux cas, la sainteté de la Normandie est avérée, ou par la sagesse de son souverain, ou par la faveur de la Vierge. Dans les deux cas également, c'est une sorte de jurisprudence qui se met en place, et qui rappelle que l'on n'est pas damné tant que le péché n'a pas été effectif. La Normandie se place comme état de droit autant que comme état de foi, et prolonge une réflexion sur le libre arbitre qui a été réactivée par le dialogue de saint Anselme sur le sujet.

La comparaison de ces deux textes, si elle est indiscutablement révélatrice d'une parenté, ne résout pas tous les problèmes : on peut bien sûr considérer que Wace connaissait l'histoire par le texte d'Eadmer, puisqu'il figure avec le miracle d'Helsin dans le Sermo de conceptione Mariae. Il faudrait alors se demander pourquoi il n'apparaît pas dans le texte de la Conception de Wace aux côtés de l'histoire d'Helsin, alors même qu'il en constitue, je crois l'avoir montré, un symétrique logique et tout à fait conforme au projet unificateur des souverains anglo-normands.

De fait, il existe un autre texte dans la Patrologie latine, conservé originellement à Jumièges et intitulé Miraculum de conceptione sanctae Mariae ${ }^{32}$ et qui rapporte la seule histoire d'Helsin. Les choses sont plus complexes, dans la mesure où ce recueil semble une version des collections anglo-normandes et comporte d'autres miracles ${ }^{33}$, mais sans doute pas celui du sacristain ${ }^{34}$. On peut donc imaginer que Wace, qui a manifestement suivi une source écrite pour rapporter le miracle d'Helsin au début de sa Conception, a pu ne pas avoir connaissance d'une version écrite et conceptionniste du sacristain; il aurait donc pu s'appuyer sur cette version orale, véhiculée par le moine repenti et

30 Roman de Rou, III, vers 510.

31 Ibid., vers 500-503.

32 Le texte est édité dans P.L., t. 159, col. 323.

33 La seule indication que j'aie pour l'instant est la note de la Patrologie : «In eodem ms. eodem charactere scripta leguntur plura alia miracula B. Mariae precibus edita, quae non majoris sunt fidei, inter haec unum est ac abbatissa sanctimonialis quae cum virginitatis et castitatis violasset propositum et praegnans B. Mariae suas preces funderet, ob ea obstetricantibus rebus angelis liberata est » (ibid.).

34 On peut supposer que les éditeurs l'auraient reproduit à nouveau s'ils l'y avaient retrouvé, comme ils l'avaient fait dans le Sermo. 
cautionnée par Richard qui atteste de la continuité de son songe et de la réalité du moine : beau récit qui peut invoquer des autorités indiscutables ${ }^{35}$.

Si cela fragilise l'hypothèse que j'ai suivie jusqu'ici d'un remploi à des fins politiques d'un miracle conceptionniste, cela pose de nouvelles questions, et implique qu'il existait, en amont de nos deux versions, une tradition orale assez forte et assez labile pour leur donner naissance. Il faudrait alors renverser la proposition, et faire de cette légende impliquant Richard sans Peur la matrice du miracle conceptionniste, inscrivant comme en filigrane cette fois-ci la puissance ducale dans la réécriture de cette version mariale. La démarche que l'on a tendance à attribuer à Wace, serviteur zélé de la cause anglo-normande, devrait alors être déplacée chez Eadmer, acclimatant à la cause mariale l'anecdote ducale et inscrivant en filigrane la figure du souverain normand dans la dévotion anglaise.

Mais ma proposition initiale est d'autant plus probable que dans la version de Benoît de Sainte-Maure - si j'en juge d'après l'index, on n'y compte que six mentions de Marie sur 45000 vers - la Vierge est invoquée à deux reprises, d'une part dans le miracle du sacristain où l'invocation est assez développée et associée à une promesse :
«Totes les a mais espondues,
Les foles fenmes a sa vie.
Moct reclaime saite Marie
"Secorez mei, qu'ore m'est mester
Tant que rentrez fusse eu moster.
Menez mei ci qu'a vostre image,
E ge serai apris e sage
D'or en avant sanz rien osfendre.
Aiz me lairei ardeir ou pendre
Que je jamais la porte pas" ${ }^{36}$.

d'autre part, dans l'épisode du diable dans l'église :

« Davant l'autel se reumilie,

Nostre dame saite Marie

Pree e remembre. Son salu

$\mathrm{Li}$ a treis feiz dit e rendu $»^{37}$.

Nous sommes certes à chaque fois dans un moment où, face au surnaturel, l'invocation à Dieu et ses saints est un recours utile ; mais Dieu n'y est pas

35 Un lecteur me fait remarquer qu'il est vain de « chercher les traces du fait divers authentique ». Ce qui m'intéresse ici est d'avoir trace probable d'une rumeur, d'une légende urbaine : c'est l'attestation d'une version qui me retient ici, pas l'authenticité d'un fait divers.

36 Benoît de Sainte-Maure, Chronique des ducs de Normandie, vers 28 013-28 019.

37 Ibid., vers 27 359-362. vers 27 359-27 362. 
sollicité, et pas davantage saint Ouen. On peut penser que Benoît, tout comme Wace, perçoit la facette mariale de cette séquence, qui serait donc antérieure à nos auteurs.

On le voit, il y a toujours, quelle que soit l'incertitude qui subsiste autour de cet épisode, une interaction entre l'élément marial et une apologie ducale. Si sa fonction est claire dans le projet apologétique conceptionniste, elle reste peutêtre à définir dans la série des anecdotes sur Richard. Françoise Laurent a déjà largement étudié ce passage de l'Histoire des Ducs de Normandie $e^{38}$, en s'attachant à la dimension trifonctionnelle des trois anecdotes sur Richard rapportées par Benoît. Les cartes sont un peu brouillées dans la version de Wace, qui donne non pas trois, mais quatre ou cinq récits différents, assez disproportionnés : si l'histoire du sacristain noyé est de loin la plus importante (175 vers), celle du diable dans l'église qui la précède et celles qui suivent - sur les amants décapités et le veneur entreprenant - sont plus courtes (respectivement 60, 50, 40 vers) ; il faudrait enfin ajouter à cela les 45 vers de l'anecdote relative à Gonnor. En termes d'économie du récit, on voit que les trois ou quatre anecdotes, regroupées, "pèsent » presque autant que celle du sacristain noyé, rassemblant 150 ou 200 vers.

On ne peut sans doute pas ramener les anecdotes rapportées par Wace à un schéma trifonctionnel, et leur cohérence est d'une autre nature, s'articulant davantage sur un mode binaire. Il me semble tout d'abord nécessaire d'inclure dans la série l'épisode de Gonnor : en effet, l'ensemble des anecdotes est comme délimité par deux affirmations parallèles, l'une à propos de Richard, qui ouvre le passage :

« Richars ama clers et clergie, chivaliers et chevalerie $"^{39}$.

l'autre à propos de Gonnor, qui le conclut :

" Gunor fu dame mult preisie, De bones murs, bien enseignee,

Clers et chevaliers honura,

Mult despendi e mult duna $»^{40}$.

38 F. Laurent, Pour Dieu et pour le roi : Rhétorique et idéologie dans l'Histoire des ducs de Normandie de Benoit de Sainte-Maure, Paris Champion 2010, p. 215 sq., ainsi que sa participation au colloque de Lyon Normes et Hagiographies, sous la direction de M. Gaillard et A. Dierkens, 4-6 octobre 2010, "Le Miracle du sacristain noyé à l'épreuve de l'histoire normande et des enjeux dynastiques ", Actes à paraître.

39 Roman de Rou, III, vers 273-274

40 Ibid., vers 651-654 
Il faut donc bien prendre en compte la continuité qu'il y a de Richard à Gonnor, aussi nobles l'un que l'autre, et leurs valeurs communes de chevalerie et de clergie : c'est l'enjeu manifeste de la série; mais ce n'est pas le seul. Si on a montré le caractère merveilleux des aventures de Richard, il faut sans doute souligner que lui-même est d'une nature particulière, lui qui ne semble pas dormir - " par nuit errout cumme par jur ${ }^{41}:$ il n'est pas seulement nyctalope, mais noctambule, et les aventures qui le concernent directement se placent majoritairement la nuit, comme s'il ne dormait pas, qu'il s'agisse de sa rencontre avec un diable (" une nuit vint a un mustier » ${ }^{42}$ ) ou de l'histoire du sacristain (" dormi aveit, mais dunc veillout ») - éléments que les autres légendes développeront, comme sa rencontre de la Mesnie Hellequin, qui se fait en pleine nuit ${ }^{43}$, ou sa découverte du pommier en pleine forêt chez Benoît. C'est à l'aube que Richard part à la chasse, c'est la nuit qu'il échange avec Gonnor : seule l'histoire du veneur violeur semble avoir lieu en plein jour - mais elle ne concerne pas immédiatement Richard.

Ces éléments pourraient faire de Richard une sorte de Mélion, cet être faé qui ignorait le sommeil. Cet aspect faé expliquerait alors sa capacité à ne pas s'émouvoir des fantômes et des créatures nocturnes. Mais, être de la nuit presque plus que du jour, Richard est cependant profondément chrétien, et c'est le point commun des deux premières histoires : son combat contre un diable et sa victoire viennent sans doute de sa pratique de prier dès qu'il rencontre une chapelle, et de prier qu'il soit à l'intérieur comme à l'extérieur. Toutefois cette dévotion est exactement celle d'un Muldumarec qui prouve son côté positif dans le lais d'Yonec, elle se présente presque comme un gage donné à Dieu et au lecteur afin d'être « du bon côté ».

Structuralement, un point semble commun à tous ces récits, une sorte de balancement binaire qui se produit à diverses reprises : Richard prie à l'extérieur comme à l'intérieur de l'église, il s'occupe de l'autel devant lui et refuse ce qui est derrière lui, il coupe en deux le démon, sort et entre à nouveau, pour reprendre ses gants - une paire. Dans l'histoire du sacristain, tout semble se résoudre à l'alternative : est-il pécheur ou innocent, damné ou sauvé ? Mais à la première partie de l'anecdote s'ajoute la visite du lendemain, qui remet en présence Richard et le moine. Les deux histoires suivantes ont lieu autour d'une chasse au cerf, et le couple décapité, homme et femme, en deux morceaux, est associé à une histoire où, après avoir fourché le cerf, c'est-à-dire tendu sa peau dans des branchages, le veneur indélicat est lui-même lancé dans une fourche d'arbre, passif et impuissant - comme le cerf fourché - jusqu'à ce qu'on vienne le descendre.

41 Ibid., vers 275.

42 Ibid., vers 289.

43 Rapportée dans Histoire des Ducs de Normandie et des rois d'Angleterre..., F. Michel (éd.), Paris, J. Renouard, 1840, II, p. 336. 
Le couple ducal enfin oppose deux situations : celle où Gonnor était la maîtresse soumise du duc, celle où elle est son épouse et son égale; tout cela se manifestant autour de la position de deux personnes dans le lit. C'est à la suite de cette séquence qu'on indique qu'elle soutient également chevalerie et clergie.

L'itinéraire esquissé par les anecdotes nous montre certes Richard comme un seigneur presque surnaturel et profondément chrétien, d'une grande vaillance comme d'une grande piété : chevalerie et clergie. Il montre ensuite deux séquences où il est question d'un couple, le premier où l'homme décapite sa compagne avant d'être tué lui-même, le second qui éclate après le viol réel ou évité. Les choses de la chair y sont également étranges et inexpliquées, et remontent à des substrats sans doute archaïques : la double référence au cerf, l'indication pour la première histoire que l'on se trouve bien après août $t^{44}$, au moment où va commencer le brame du cerf, tout cela surdétermine une dimension sexuelle symbolique dans un premier temps, et très explicite dans la deuxième histoire ${ }^{45}$. Dans les deux cas, nous sommes également confrontés à l'inexplicable d'une femme décapitée, d'un amant projeté dans les airs.

Ces situations renvoient à une sexualité violente et sans loi, où Richard est un justicier en même temps qu'un criminel en ce qu'il tue cet homme meurtrier de la femme sans rien savoir de ce qui s'est déroulé. La dernière histoire, où il n'intervient pas, semble juste appelée par sa parenté de lieu et de temps avec la précédente ; ce n'est plus en apparence la figure du souverain qui légitime le propos, mais la violence de la relation, violence aussi étrange et peu maîtrisée que celle du diable dans la chapelle, mais centrée sur la dimension sexuelle. Autant qu'il le peut, Richard s'efforce de mettre les choses en ordre, de les racheter ou de les réguler, d'instaurer un mode de vie raisonnable là où règnent chaos et violence.

Face à cela, le mariage de Richard devient une réponse essentielle, en ce que l'on quitte l'arbitraire du concubinage pour entrer dans l'ordre d'un mariage officiel; mariage d'autant plus symbolique qu'il concerne une princesse née « de nobles Daneis ${ }^{46}$ : alors qu'une union more danico est comme évidente pour deux lignages de même origine, le mariage chrétien devient un engagement d'un nouveau type, engagement béni de surcroît par une belle descendance.

Il n'est pas indifférent que le mariage soit annoncé comme issu de la demande pressante et du clergé et de l'aristocratie, relevant de ce fait de la chevalerie et de la clergie que l'on a déjà relevées :

44 « ja iert de aüst li meis passez» (Roman de Rou, III, vers 517).

45 Ce n'est pas le lieu ici de rappeler la dimension symbolique du cerf et de ses ramures dans la tradition occidentale, de Cernunnos à Falstaff.

46 Roman de Rou, III, vers 239. 


\begin{abstract}
«Mais par preiere del clergié
Ke l'en out meinte feiz preié

E par cunseil de ses baruns,

Qui mainte feiz l'en unt somuns,

Ad Ricard Gunnor espusee ${ }^{47}$.
\end{abstract}

Le monde de Richard instaurait, les deux premières anecdotes l'ont montré, un monde de chevalerie et de clergie, un monde de force et de sagesse, intégré dans l'ordre divin, et qui aboutit régulièrement à des règles et des recommandations à l'usage de tous. Richard ordonne que les morts soient veillés désormais, même dans les églises ; les moines savent maintenant qu'il ne faut pas sauter le pas ou passer la planche ; il semble dans cette perspective que le mariage ducal, more christiano, est une réponse d'ordre, de loi et de sagesse aux étrangetés d'un amour libre qui aboutit à la mort des amants ou à la projection dans un arbre, qui reconnaît la pulsion amoureuse comme dangereuse, la femme comme inquiétante et comme vulnérable. Si certaines précipitent leur amant à la fourche d'un arbre, Gonnor avoue elle-même en parlant du lit désormais conjugal :

«Unkes mais aseüre n’i jui,

$\mathrm{Ne}$ sanz poür od vus ne fui,

Ore sui aukes aseüree ${ }^{48}$.

Ces vers constituent une réponse au cri de Richard, quand il se précipite sur le chevalier pour l'empêcher de décapiter la dame :

«Li quens cria : "mal faiz, mal faiz!

Femme deit aueir partut paiz" " ${ }^{49}$.

S'il se sent coupable à l'égard du chevalier décapité - cette histoire est de celles que seule la tradition orale rapporte - c'est peut-être parce que Richard avant son mariage religieux est au bout du compte aussi dangereux pour une femme que ce qu’a pu être le chevalier. Ce qui est donc en jeu dans ces épisodes, c'est certes chevalerie et clergie, vaillance et sagesse partagées par le duc et la duchesse. Cependant, c'est encore bien davantage une mise en ordre juridique et légale du monde : il n'y a plus d'indicible, de mystérieux, mais une société en place régie par des règles claires et que l'on peut exprimer. La mémoire garde trace d'un seigneur qui a réglementé les choses, même s'il reste coupable d'avoir tué un chevalier qui avait décapité une dame. Il a fait justice sur lui-même également, en reconnaissant Gonnor et en lui permettant de ne plus avoir peur dans son propre lit. C'est ce que souligne la multiplicité des références à des autorités, écrites comme orales, témoignant de la sagesse reconnue d'un

47 Ibid., vers 615-619.

48 Ibid., vers 643-645.

49 Ibid., vers 545-546. 
seigneur dont la chevalerie comme la clergie semblent dépasser l'entendement du temps. Peut-être après tout faut-il ajouter foi aux exclamations qui scandent la chronique de Dudon, comme si Richard ne pouvait être qu'un être exceptionnel digne des plus enthousiastes épithètes.

À cette mise en ordre, more christiano, d'une société qui peinait à trouver ses lois et sa paix intérieure, il convient d'ajouter, pour finir, un dernier élément : on sait que la dénonciation de la forêt de Brocéliande intervient chez Wace juste avant le récit de la bataille d'Hastings, et plus précisément avant que soient évoquées la comète et les reliques confiées au duc de Normandie. Wace, consciemment, dévalorise un merveilleux breton païen pour instaurer un merveilleux normand chrétien ${ }^{50}$. J'ai le sentiment que l'enjeu est le même ici : on a avec ce mort dans l'église dont s'empare un diable un épisode bien proche du roman certes plus tardif de l'Âtre périlleux; on retrouve dans ce goût pour la décapitation quelque chose des beheading games qui se multiplient dans le Perlesvaus ou dans Sir Gawayne and the Grene Knight. Tout se passe somme toute comme si l'on disqualifiait là aussi quelque chose qui est du registre d'un merveilleux celtique pour valoriser un merveilleux chrétien et normand, un état de loi plus qu'un état de coutume ; comme si l'enjeu n'était pas tant de passer d'une celtité à une normannité que de la barbarie incontrôlée à un monde où le souverain est également le garant de l'Église et d'une société harmonieuse.

Quelles conclusions tirer de cette enquête ? L'absence d'une dévotion mariale dans la vie de Richard, si elle est éclatante, laisse cependant affleurer, dans la version qu'en donnent Wace et Benoît, comme un arrière-plan qui n'est pas tant à vrai dire marial qu'identitaire. Cette piste permet de comprendre différemment l'organisation des épisodes, qui prennent en interrelation une signification plus claire, valorisant non seulement la chevalerie et la clergie du duc, mais également celle du couple : Gonnor est parée des mêmes vertus que son époux devant Dieu. Entre le temps de l'histoire et celui de l'écriture, ce qui est mis en place, au détriment d'un merveilleux arthurien et d'un amour libre, c'est un univers de foi, de loi et d'union conjugale : un ordre normand idéal qui maîtrise autant le monde des hommes qu'il est reconnu et respecté dans l'autre monde, où anges et démons se soumettent au jugement du duc. C'est tout l'enjeu que développe le Jeu d'Adam contemporain et normand, qui insiste sur l'union d'Adam et Eve en termes d'égalités1.

50 D. HüE « Présence des Bretons dans quelques chroniques normandes », dans Bretons et Normands au Moyen Âge, Rivalités, malentendus, convergences, Rennes, Presses universitaires de Rennes « Histoire ", 2008, p. 281-300.

51 Le Jeu d'Adam, V. Dominguez (éd. et trad.), Paris, Champion Classiques, 2012, vers 12 sq. 
Mais c'est également l'enjeu de ce qui, pas très loin, dans le diocèse de Rennes puis en Anjou, se développe avec Robert d'Arbrissel ${ }^{52}$, de ce que véhiculeront bientôt les poètes d'Oc. Cette émergence de la culture courtoise, d'une nouvelle façon de voir les femmes - au nombre desquelles la Vierge a une place exemplaire-, ne faut-il pas également en discerner les prémices dans la légende de Richard sans Peur?

\title{
Résumé
}

Sans la Vierge, Richard sans Peur et la femme. - L'enquête sur la présence mariale dans les premières biographies de Richard sans Peur tourne court, car on lui attribue un des principaux miracles évoqués à l'origine de la fête de la Conception; cet article s'efforce de montrer comment, au-delà de l'épisode du sacristain noyé dont on dégage les implications, les anecdotes autour de Richard mettent en place les éléments d'une civilisation, juridique, religieuse, où la position de la femme prend une nouvelle stature.

Mots-clefs : Wace - Benoît de Sainte-Maure - Dudon de Saint-Quentin - Conception de Marie - anecdotes - sacristain noyé.

\begin{abstract}
Without the Virgin - Richard the Fearless and Women. - The presence of the Virgin Mary in the first biographies of Richard I is inconclusive since it concerns her agency in miracles during the Feast of the Conception. This article hopes to show that, beyond the anecdote of the drowned sacristan, these stories renewed conceptions of civilisation and the civil, religious and legal role of women.
\end{abstract}

Key words : Wace - Benoît of Saint-Maure - Dudon de Saint-Quentin - the Conception of the Virgin - anecdotes - sacristans - drowning.

52 J. Dalarun, "Robert d'Arbrissel et les femmes », Annales. Économies, Sociétés, Civilisations, 1984, vol. 39, nº, p. 1140-1160. 